\title{
An Early Periprosthetic Fracture of a Cementless Oxford Unicompartmental Knee Arthroplasty: Risk Factors and Mitigation Strategies
}

\author{
Li Zong Xian ${ }^{1}$, Andrew Tan Hwee Chye ${ }^{1}$
}

Learning Point of the Article:

Early identification of high-risk patients and a careful surgical technique can prevent early periprosthetic fractures in the Oxford unicompartmental knee arthroplasty.

\section{Abstract}

Introduction: The cementless Oxford partial knee arthroplasty is associated with low perioperative complications and good long-term survival rates. However, perioperative fractures remain a serious morbidity for patients.

Case Report: This case report describes an early post-operative tibial periprosthetic fracture through the keel slot, which we believe may be contributed by the deep implant keel design and the presence of a narrow metaphysis in the Asian knee. The patient subsequently underwent a revision total knee replacement and fixation of the periprosthetic fracture.

Conclusion: This paper discusses the ways to identify patients at high risk of developing periprosthetic fractures and to minimize such occurrences, including adopting a modified tibial preparation, doing precise saw cuts, and considering a cemented tibial implant.

Keywords: Cementless, Oxford, unicompartmental, periprosthetic, keel.

\section{Introduction}

Unicompartmental knee arthroplasty (UKA) is a common and effective surgical treatment for end-stage unicompartmental knee osteoarthritis, amounting to about $5-10 \%$ of all knee arthroplasties done $[1,2,3]$. They have numerous advantages over total knee arthroplasty (TKA), including being a less invasive procedure, having less operative blood loss, shorter recovery time, lower complication rates, superior patientreported outcomes, and reduced mortality $[4,5]$. In the New Zealand Joint Registry, the cementless Oxford UKA (OUKA) has a $96 \% 10$-year survival which is similar to that achieved by TKA [6].

Despite the numerous advantages, periprosthetic medial tibia plateau fractures form a relatively rare yet potentially serious complication in patients undergoing UKA [7]. Earlier literature reported the incidence of such fractures to be as high as 5\% [8].
Periprosthetic tibial plateau fractures in cementless UKA are a potential complication of UKA that usually presents in the perioperative period. This usually occurs as a fracture line propagating at the lateral tibia tray bone junction due to a stress riser from an excessive sagittal cut [9]. Other mechanisms include propagation from the keel slot when the keel slot is too small, requiring forceful impaction for component insertion [10]. Known risk factors include damage to the posterior cortex [9] and usage of multiple pins during tibial cutting block fixation [11]. Although the published incidence of tibial plateau fractures after cementless OUKA is low, we are aware of reports of some surgeons having a higher incidence $[7,8,12]$.

This case report describes a periprosthetic fracture of the medial tibia plateau propagating from the keel slot. This rare but catastrophic early post-operative complication can potentially be prevented with good patient selection and a careful surgical technique.

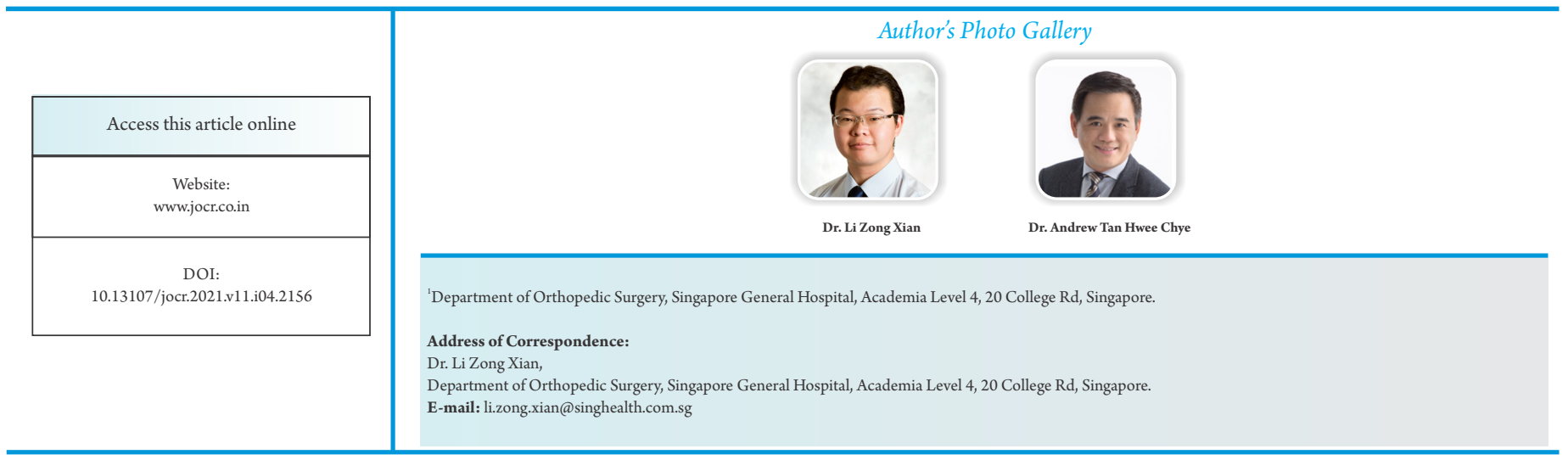

Journal of Orthopaedic Case Reports | pISSN 2250-0685 | eISSN 2321-3817 | Available on www.jocr.co.in | doi:10.13107/jocr.2021.v11.i04.2156 This is an Open Access article distributed under the terms of the Creative Commons Attribution Non-Commercial License (http://creativecommons.org/licenses/by-nc/3.0) which permits unrestricted non-commercial use, distribution, and reproduction in any medium, provided the original work is properly cited. 


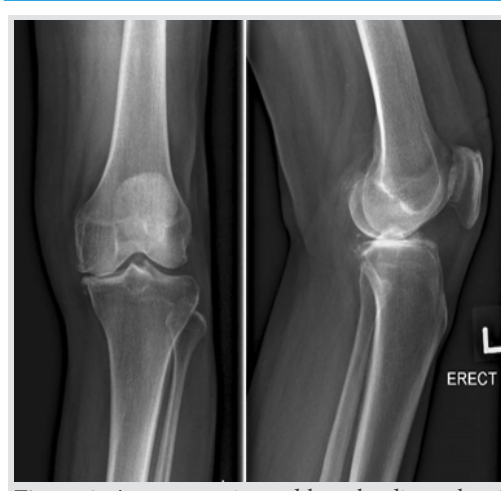

Figure 1: Anteroposterior and lateral radiographs of the pre-operative knee.

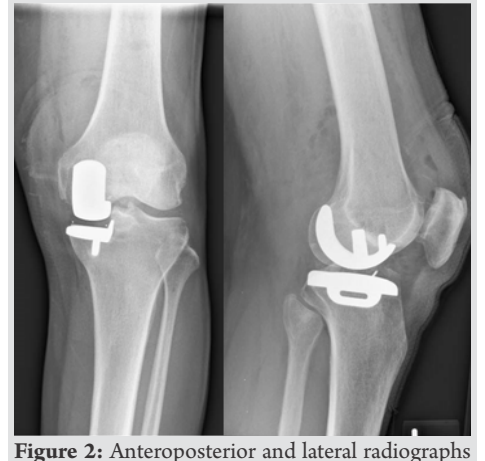

Figure 2: Anteroposterior and lateral radiograph of the post-operative knee after Oxford unicompartmental knee arthroplasty.

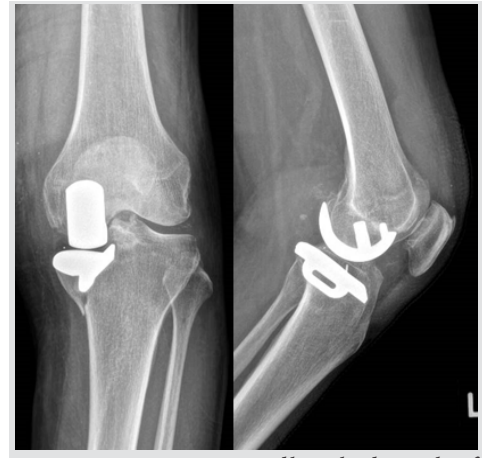

Figure 3: Anteroposterior and lateral radiographs o the periprosthetic fracture following after the onset of worsening ambulatory pain.

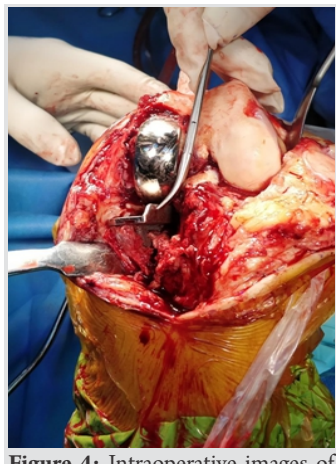

Figure 4: Intraoperative images of the tibial periprosthetic fracture through the keel slot.

\section{Case Report}

An 80-year-old Chinese lady with symptomatic left medial knee osteoarthritis confirmed on weight-bearing knee radiographs (Fig. 1) presented to our outpatient clinic.

Having failed a period of conservative treatment, she subsequently underwent a left medial OUKA successfully. A cementless size XS femoral implant and a cementless size AA tibial implant with a $5 \mathrm{~mm}$ mobile bearing insert were used. Post-operative recovery was uneventful, and radiographs done showed satisfactory prosthesis positioning with no evidence of periprosthetic fractures (Fig. 2). The patient managed to ambulate well on the 1st post-operative day and was transferred to a step-down facility for further rehabilitation.

The patient underwent further therapy in the step-down facility and showed good progress and recovery with tolerable levels of knee discomfort. However, 2 weeks on, she started complaining of worsening knee pain with decreased ambulatory distances while still being able to weight bear and ambulate. There were no preceding instances of trauma or injury. A repeat plain radiograph showed a displaced proximal tibial periprosthetic fracture (Fig. 3). She was immediately placed on a non-weightbearing status and transferred back to our hospital for further management. A pre-operative computed tomography (CT) scan was deemed unnecessary as the periprosthetic fracture was

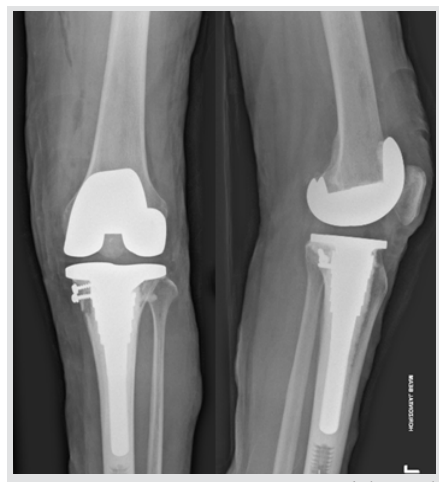

Figure 5: Anteroposterior and lateral radiographs of the revision total knee arthroplasty.

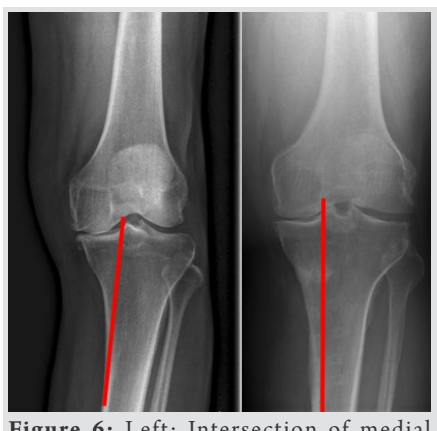

Figure 6: Left: Intersection of medial eminence line along tibial long axis just medial to the medial tibial eminence with the medial diaphyseal cortex indicates that cemented tibial trayshould be considered.

of a simple fracture pattern and clearly defined by the orthogonal plan radiographs.

She was counseled and underwent left revision TKA with fixation of the tibial plateau fracture. Intraoperative findings include a medial tibial periprosthetic fracture with the fracture originating from the keel slot of the cementless tibia implant (Fig. 4). The implants were revised to a cemented size 2.5 Depuy Synthes Press Fit Condylar femur and size 2 Mobile Bearing Tibia tray and $29 \mathrm{~mm}$ femur sleeve (Fig. 5). Postoperative full weight-bearing ambulatory physiotherapy was permitted and tolerated well. A dual-energy X-ray absorptiometry bone mineral densitometry, performed to assess the density of the bone architecture as a risk factor for the periprosthetic fractures, showed osteopenia.

On discharge, the patient was reviewed regularly in the outpatient clinic and at the latest review at 6 months postsurgery, she was noted to be walking well with satisfactory range of motion of $15-95^{\circ}$.

Verbal consent was obtained for the patient for the purpose of this case report.

\section{Discussion}

Our case study describes a periprosthetic fracture of the medial tibia plateau as a post- operative complication after OUKA. The fracture is unique in that it occurred through the keel slot rather

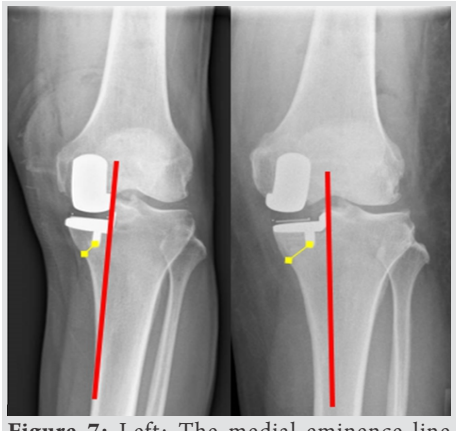

Figure 7: Left: The medial eminence line extrapolated from the vertical tibial cut intersecting the medial diaphyseal cortex, demonstrating a small bone bridge. Right: A nonintersecting line showing a larger bone bridge.

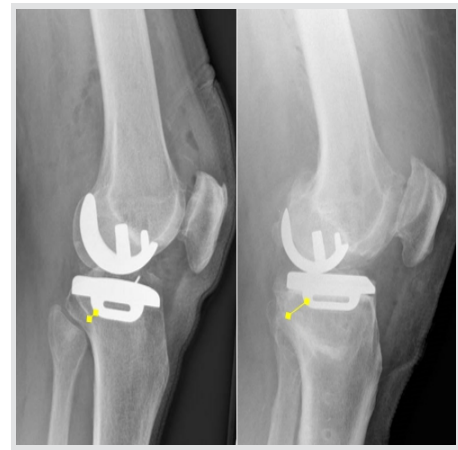

Figure 8: Differing posterior metaphyseal cortex morphology, demonstrating a much narrower posterior bone bridge in our patient's Oxford unicompartmental knee arthroplasty on the left. 
than along the lateral bone-implant junction. We believe that this may have started as a stress fracture intraoperatively and worsened with time. It explains why the patient was initially walking well with minimal pain.

It has been shown in previous anthropometric studies that the Asian knee morphology differs from the Caucasian knee in terms of size and shape, with a notably smaller and narrower proximal tibia metaphysis $[13,14]$. The Oxford partial knee system tibial component also demonstrated a mediolateral mismatch to measured anteroposterior resections in up to $71.3 \%$ of Chinese knees [15]. It is also likely that the exceptionally deep keel and this smaller bone bridge between the lowest point of the keel slot and the metaphyseal cortex may have resulted in an increased risk of stress fractures.

The medial eminence line (MEL), a vertical line parallel to the tibial axis drawn through the medial intercondylar eminence, has been described. If this line intersects with the medial diaphyseal cortex, the risk of post-operative stress fractures will be increased $[16,17]$. In such cases, a cemented tibial component should be considered to increase the loading capacity and dissipate the stresses around the cement mantle [9] (Fig. 6).

This concept can be further extrapolated to the evaluation of the post-operative radiograph, where the MEL coincides with the lateral most border of the tibial implant (Fig. 7). If this line intersects with the medial diaphyseal cortex inferiorly, it usually means that the distance between the tip of the keel and medial tibial metaphyseal cortex is decreased, with a higher risk of fracture propagation. In such patients, a high index of suspicion for periprosthetic fracture should be had when they present with post-operative ambulatory pain, and a more cautious weight-bearing regime should be adopted.

It is also recommended that a more lateralized tibial tray position should be aimed for in the medial OUKA to achieve a larger tibial bone bridge between the keel and the metaphyseal cortex. Often, the vertical tibial bone cut may be erroneously positioned at the base of the medial tibial eminence when it should be positioned instead further lateral just medial to the footprint of the ACL fibers at the apex of the medial tibial eminence. This may result in an overly medialized tibial tray and excessively narrow bone bridge beneath it, greatly increasing the risk of perioperative fracture.

Very often, intraoperative and early post-operative periprosthetic tibial plateau fractures are due to technical errors [7]. Clarius et al. have shown that lifting the saw hand and extending the sagittal cut by as little as $10^{\circ}$ can reduce the loading capacity by as much as $30 \%$ [18]. Seeger et al. have shown that such periprosthetic risks may be mitigated using a cemented tibia tray fixation, but great attention must still be given during the sagittal cuts to avoid lifting the saw hand and extending the cuts [9].

It is also noted that the anteroposterior diameter of the proximal tibial metaphysis in our Asian knees tends to be narrower and has a more conical morphology. This can often lead to an exceptionally small posterior tibial bone bridge between the posterior-most portion of the keel and the posterior tibial metaphyseal cortex (Fig. 8). This can potentially result in a stress riser due to the narrower bone bridge, leading to a fracture line propagating into the posterior cortex. Similarly, during the preparation of the keel slot or insertion of the tibial implant, the narrow bridge may also lead to an inadvertent breach of or stress to the posterior metaphyseal cortex.

During insertion of the cementless tibial component, the keel slot is often prepared smaller than the actual implant keel to allow for press fit insertion and greater pull-out strength. The impaction forces required to mallet the implant flush with the bone can be as high as $1400 \mathrm{~N}$, which can stress the bone and even result in fractures $[19,20,21]$. To prevent this, we propose placing the tibial implant directly over the tibial slot and using a light mallet to hit gently inferiorly, as opposed to hitting the implant posteroinferiorly to seat it in the slot, which may stress the posterior tibial bone bridge.

With the above, tibial keel related factors form a significant risk for periprosthetic fracture in the cementless OUKA system. This can be narrowed down to the larger keel size as well as mismatch of the keel slot preparation and implant keel size $[10$, 16].

Mohammad et al. suggested modifications to the traditional keel preparation saw blade to reduce the risk of perioperative tibial fractures. In the new design, the blade was $0.2 \mathrm{~mm}$ wider at the tip and $0.7 \mathrm{~mm}$ deeper. This was shown to halve the push-in forces without significantly affecting the pull-out forces. Reduction of push-in forces may also be achieved using the cemented preparation pick to widen the slot slightly while preparing for a cementless tibia tray. However, this may potentially reduce the fixation strength by reducing the pull-out forces [18]. These methods are recommended if the risk of periprosthetic fractures is determined to be high, for instance in patients with osteoporotic bone.

If much resistance is experienced during impaction, the option to leave the tibia tray slightly proud should be considered. The tray will undergo controlled subsidence subsequently as the patient ambulates and the surrounding bone undergoes stress relaxation.

Postoperatively, if increasing pain is experienced through the course of ambulatory physiotherapy, immediate reduced weight-bearing should be advised to prevent an intraoperative stress injury or fracture from propagating into a complete 
fracture.

The role of advance imaging techniques like CT scans should be considered if there are doubts or suspicions for a periprosthetic fracture in a patient presenting with increasing ambulatory pain. This can also be useful to look for excessive sagittal cuts or breach in the posterior tibial cortex so that the patient in be put on a period of protected weight-bearing to avoid a periprosthetic fracture.

The "take-off" sign has been described when early medial shifting of the tibial component is seen in serial radiographs with minor separation between the bony cuts and component surfaces [16]. This should alert the surgeon to the significant possibility of a subsequent complete periprosthetic fracture.

In this case report, the periprosthetic medial tibial fracture has rendered the implant unstable as seen by the loosening and migration on the post-fracture radiograph. An option for internal fixation with restoration of the implant positioning can be considered. However, a period of non-weight-bearing is required for fracture healing and may negatively affect the arthroplasty outcomes. Furthermore, with the pre-existing anatomical issue of the narrow bone bridge and decreased volume of supporting bone beneath the tibial implant, the risk of recurrence and refracture will be significant. Therefore, we opted to revise this UKA into a TKR with fixation of the medial tibial fracture fragment. A stemmed tibial implant was used as well to offload the fractured medial tibial metaphysis onto the diaphyseal cortex instead. This will aid in fracture healing as well as allow immediate post-operative full weight-bearing.

The decision for internal fixation versus revision to TKR in such a fracture should hinge on the likely mechanism and cause of the early periprosthetic fracture. If the reason for failure is deemed to be from technical errors like over cutting of the sagittal saw cut or breach of the posterior cortex, then fixation with protected weight-bearing until fracture union can be attempted. If the cause of failure is due to anatomical reasons like a conicalshaped tibial metaphysis with an overhanging tibial plateau or an intersection MEL, then a revision to a more stable construct like a TKR should be considered.

\section{Conclusion}

Medial tibia plateau periprosthetic fractures can occur after cementless OUKA. Risks are higher in osteoporotic patients, and patients with more conical metaphyses and smaller bone bridges after tibial keel slotting are done. Identification of these patients is important, and the various methods and modifications as described are key to preventing such a complication.

\section{Clinical Message}

Patients at high risk of periprosthetic OUKA fracture can be identified easily and mitigative action can be carried out intraoperative to prevent such catastrophic failures.

\section{References}

1. Canadian Joint Replacement Registry Report: Total Hip and Total Knee Replacements in Canada: Executive Summary. Canada: Canadian Joint Replacemet Registry; 2006.

2. Australian Orthopaedic Association. National Joint Replacement Registry. Annual Report. Adelaide: Australian Orthopaedic Association; 2006.

3. The Swedish Knee Arthroplasty Register, Annual Report $2006 ; 2006$.

4. Liddle AD, Judge A, Pandit H, Murray DW. Adverse outcomes after total and unicompartmental knee replacement in 101,330 matched patients: A study of data from the National Joint Registry for England and Wales. Lancet 2014;384:1437-45.

5. Goodfellow J, O’Connor J, Murray DW. The Oxford meniscal unicompartmental knee.J Knee Surg 2002;15:240-6.

6. The New Zealand Joint Registry. Seventeen Year Report January 1999 to December 2015. New Zealand: New Zealand Joint Registry; 2016.
7. Pandit H, Murray DW, Dodd CA, Deo S, Waite J, Goodfellow J, et al. Medial tibial plateau fracture and the Oxford unicompartmental knee. Orthopedics 2007;30:28-31.

8. Rudol G, Jackson MP, James SE. Medial tibial plateau fracture complicating unicompartmental knee arthroplasty. J Arthroplasty 2007;22:148-50.

9. Seeger J, Haas D, Jäger S, Röhner E, Tohtz S, Clarius M. Extended sagittal saw cut significantly reduces fracture load in cementless unicompartmental knee arthroplasty compared to cemented tibia plateaus: An experimental cadaver study. Knee Surg Sports Traumatol Arthrosc 2012;20:1087-91.

10. Van Loon P, de Munnynck B, Bellemans J. Periprosthetic fracture of the tibial plateau after unicompartmental knee arthroplasty. Acta Orthop Belg 2006;72:369-74.

11. Brumby SA, Carrington R, Zayontz S, Reish T, Scott RD. Tibial plateau stress fracture: A complication of unicompartmental knee arthroplasty using 4 guide 
pinholes.J Arthroplasty 2003;18:809-12.

12. Berger RA, Meneghini RM, Jacobs JJ, Sheinkop MB, Della Valle CJ, Rosenberg AG, et al. Results of unicompartmental knee arthroplasty at a minimum of ten years of follow-up. J Bone Joint Surg Am 2005;87:9991006.

13. Zengin A, Pye S, Cook M, Adams J, Wu F, O’Neill T, et al. Ethnic differences in bone geometry between White, Black and South Asian men in the UK. Bone 2016;91:180-5.

14. Kim TK, Phillips M, Bhandari M, Watson J, Malhotra R. What differences in morphologic features of the knee exist among patients of various races? A systematic review. Clin Orthop Relat Res 2017;475:170-82.

15. Lu F, Zhang Q, Liu P Guo W. Anthropometry of the medial tibial plateau in the Chinese population: The morphometric analysis and adaptability with Oxford Phase III tibial components. Knee Surg Sports Traumatol Arthrosc 2020;28:3048-54.

16. Hiranaka T, Yoshikawa R, Yoshida K, Michishita K, Nishimura T, Nitta $S$, et al. Tibial shape and size predicts the risk of tibial plateau fracture after cementless unicompartmental knee arthroplasty in Japanese patients.

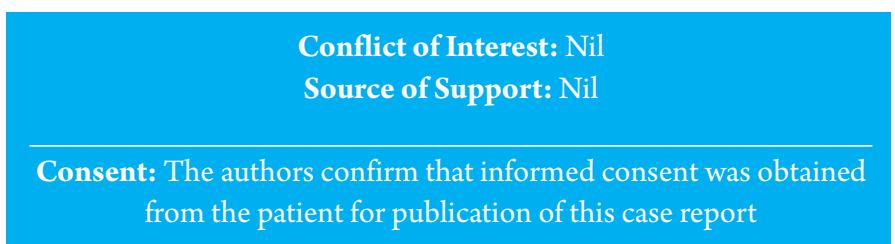

Bone Joint J 2020;102-B:861-7.

17. Yoshikawa R, Hiranaka T, Okamoto K, Fujishiro T, Hida YS. The Medial Eminence Line for Predicting Tibial Fracture Risk after Unicompartmental Knee Arthroplasty. Clin Orthop Surg 2020;12:166-70.

18. Clarius M, Haas D, Aldinger PR, Jaeger S, Jakubowitz E, Seeger JB. Periprosthetic tibial fractures in unicompartmental knee arthroplasty as a function of extended sagittal saw cuts: An experimental study. Knee 2010;17:57-60.

19. Mohammad HR, Campi S, Murray D, Mellon S. Instruments to reduce the risk of tibial fracture following cementless unicompartmental knee replacement. Knee 2018;25:988-96.

20. Damm NB, Morlock MM, Bishop NE. Friction coefficient and effective interference at the implant-bone interface. J Biomech 2015;48:3517-21.

21. Nowak M, Kusz D, Wojciechowski P, Wilk R. Risk factors for intraoperative periprosthetic femoral fractures during the total hip arthroplasty. Pol Orthop Traumatol 2012;77:5964.

\section{How to Cite this Article}

Li ZX, Tan HCA. An Early Periprosthetic Fracture of a Cementless Oxford Unicompartmental Knee Arthroplasty: Risk Factors and Mitigation Strategies. Journal of Orthopaedic Case Reports 2021 April;11(4):65-69. 